\title{
Atypical Clinical Presentation and Imaging Findings of Central Nervous System Tuberculosis
}

\author{
Manav V. Vyas, Paul E. Bunce, Tim-Rasmus Kiehl, Mark Bernstein, \\ Richard Wennberg
}

Keywords: Acute disseminated encephalomyelitis (ADEM), Apraxia, Gerstmann syndrome, Magnetic resonance imaging (MRI), Tuberculosis

doi:10.1017/cjn.2018.356

Can J Neurol Sci. 2018; 45: 686-687

A 70-year-old man of South-Asian descent with a past medical history of benign prostatic hypertrophy, type-2 diabetes mellitus, hypertension and gastro-oesophageal reflux presented with a onemonth history of falls, confusion and bilateral hand paraesthesia. There was no prodromal illness. On examination, he was unable to calculate or finger count and had right-left confusion, compatible with a partial Gerstmann syndrome. ${ }^{1}$ He could not perform simple motor tasks to command (ideomotor apraxia), had diffusely brisk reflexes and gait apraxia. MRI brain showed biparietal white matter T2-hyperintensities not enhancing after gadolinium (Figures 1A, 1B). CSF examination disclosed two white blood cells, normal protein, normal glucose and positive oligoclonal bands. CT chest-abdomen-pelvis revealed no signs of malignancy and a few lung nodules that were not amenable to needle biopsy. Image-guided brain biopsy revealed a reactive infiltrate with marked gliosis and perivascular macrophage aggregation (Figures 1C, 1D). Additional Ziehl-Nelson staining showed no organisms. Detailed immunochemistry for infectious aetiologies such as toxoplasmosis, promyelocytic leukaemia, herpes simplex virus (HSV)-1 or HSV-2 and cytomegalovirus was negative. Tuberculosis skin test was positive $(14 \mathrm{~mm})$; the patient and family declined treatment for latent tuberculosis given poor prognosis. On the basis of initial investigations, a decision was made to treat with intravenous corticosteroids $(1 \mathrm{~g}$ methylprednisolone for 5 days) followed by oral prednisone taper for a presumptive diagnosis of atypical acute disseminated encephalomyelitis (ADEM), given the absence of definite infectious or neoplastic causes and the presence of CSF oligoclonal bands. The patient experienced some clinical improvement in ability to ambulate and he was discharged to inpatient rehabilitation, although the post-treatment MRI scan did not show significant improvement (Figures 1E, 1F).

One week later, the brain biopsy specimen culture returned positive for Mycobacterium tuberculosis. The patient was started on a 12-month course of treatment with isoniazid, rifampin, pyrazinamide, ethambutol and pyridoxine.

M. tuberculosis leads to central nervous system (CNS) involvement in about $1 \%$ of all cases (80,000 cases studied) in Canada. $^{3}$ Risk factors for CNS involvement include children, HIV-infected individuals, malnutrition, alcoholism, immunosuppression and immigration status. ${ }^{2}$ The pathogenesis of CNS tuberculosis is not fully understood, but is believed to result from haematogenous dissemination of mycobacteria in the brain, where a delayed immune recognition or reactivation leads to development of clinical manifestations. ${ }^{3}$ The most common presentations of CNS tuberculosis include tubercular meningitis, intracranial tuberculoma or abscess, and Pott's disease. ${ }^{4}$

A rare presentation of CNS tuberculosis in the form of encephalopathy was first described in infants and children by Udani et $\mathrm{al}^{5}{ }^{5}$ who reported a diffuse cerebral disorder that included convulsions, stupor and coma without focal neurological deficits. CSF analysis in these patients was either normal or showed high protein and white cell count. ${ }^{5}$ The pathophysiology of this presentation is not well understood but is felt to represent an aberrant immune response to the mycobacterium in hosts with an immature immune system. ${ }^{5}$ Some of the reported patients, similar to this case, presented with a clinical picture resembling ADEM ${ }^{5,6} M$. tuberculosis should be included in the differential diagnosis for an ADEM-like presentation, especially in patients with consistent exposure history or risk factors.

\section{DisClOSURE}

MVV, PB, TRK, MB and RW have nothing to disclose.

\section{Statement of Authorship}

MVV, PEB, TRK, MB and RW were involved in conception and design of the study. MVV drafted the manuscript, which was critically revised for important intellectual content by all authors.

From the Division of Neurology, Department of Medicine, University of Toronto, Toronto, Ontario, Canada (MVV, RW); Division of Infectious Diseases, Department of Medicine, University of Toronto, Toronto, Ontario, Canada (PEB); Department of Laboratory Medicine and Pathology, University of Toronto, Toronto, Ontario, Canada (TRK); Division of Neurosurgery, Department of Surgery, University of Toronto, Toronto, Ontario, Canada (MB)

Received June 1, 2018. Date of Acceptance September 8, 2018.

Correspondence to: Manav V. Vyas, Division of Neurology, University of Toronto, 2075 Bayview Avenue, A455, Toronto, ON, Canada M4N 3M5. Email: manav.vyas@mail. utoronto.ca 

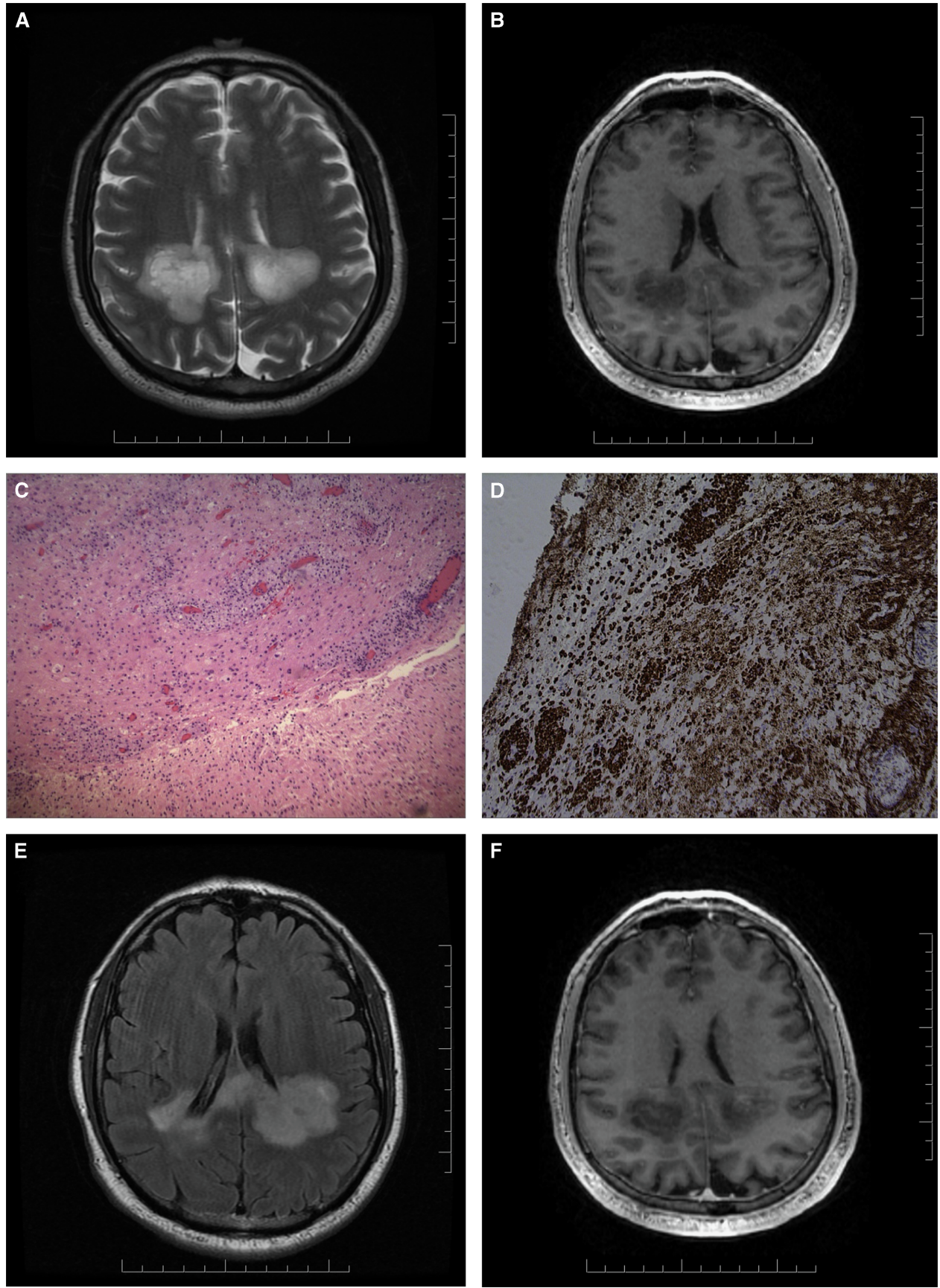

Figure 1: Axial T2 MRI brain (A) and T1-weighted contrast study (B) show bilateral, left more than right, white matter predominant lesions with involvement of the splenium of the corpus callosum. Haematoxylin and eosin stains show mixed inflammatory infiltrate with marked gliosis and no mitosis or necrosis (C). Perivascular macrophages are seen with CD-68 stain (D). Axial T2 FLAIR (E) and T1-weighted contrast study $(F)$ show no significant improvement post steroid treatment.

\section{REFERENCES}

1. Vallar G. Spatial neglect, Balint-Homes' and Gerstmann's syndrome, and other spatial disorders. CNS Spectr. 2007;12(7):527-36.

2. Phypers M, Harris T, Power C. CNS tuberculosis: a longitudinal analysis of epidemiological and clinical features. Int $\mathrm{J}$ Tuberc Lung Dis. 2006;10(1):99-103.

3. Be NA, Kim KS, Bishai WR, Jain SK. Pathogenesis of central nervous system tuberculosis. Curr Mol Med. 2009;9(2):94-9.
4. Garg RK. Tuberculosis of the central nervous system. Postgrad Med J. 1999;75(881):133-40.

5. Udani PM, Dastur DK. Tuberculous encephalopathy with and without meningitis. Clinical features and pathological correlations. J Neurol Sci. 1970;10(6):541-61.

6. Masoodi I, Farooq O, Ahmad I, et al. Acute disseminated encephalomyelitis as the first presentation of CNS tuberculosis: report of a case with brief review. Ger Med Sci 2010;8: Doc 32. https://doi. org/10.3205/000121. 